\title{
RSRC1 suppresses gastric cancer cell proliferation and migration by regulating PTEN expression
}

\author{
SHIJUN YU*, NISHIM GAUTAM*, MING QUAN and YONG GAO \\ Department of Oncology, Shanghai East Hospital, Tongji University School of Medicine, Shanghai 200120, P.R. China
}

Received December 11, 2018; Accepted May 10, 2019

DOI: $10.3892 / \mathrm{mmr} .2019 .10409$

\begin{abstract}
Arginine/serine-rich coiled coil 1 (RSRC1) is a gene which plays a significant role in the constitutive and alternative splicing of mRNA and transcriptional regulation. It has been implicated in various neurological disorders, as well as in cancer. However, its role in gastric cancer (GC) remains unknown. Thus, the present study aimed to investigate the role of RSRC1 in GC. RSRC1 expression in GC tissues was determined by RT-qPCR and immunohistochemical staining. The effects of RSRC1 on cell proliferation and migration were detected using a Cell Counting Kit-8 assay, 5-ethynyl-2'-deoxyuridine (EdU) incorporation assay and a Transwell migration assay. Western blot analysis and RT-qPCR were used to explore the molecular mechanisms of of action of RSRC1 in GC. The results indicated that RSRC1 expression was downregulated in GC tissues compared to paired normal tissues and the reduced expression of RSRC1 was shown to contribute to a poor prognosis of patients with GC. RSRC1 knockdown promoted the proliferation and migration of GC cells. In addition, the knockdown of RSRC1 decreased the expression of phosphatase and tensin homolog deleted on chromosome 10 (PTEN), a potent tumor suppressor gene controlling cellular growth and viability. On the whole, the findings of the present study indicate that RSRC1 functions as a tumor suppressor gene in GC and that it may exert its effects by regulating PTEN expression.
\end{abstract}

\section{Introduction}

Gastric cancer (GC) is a leading cause of cancer-related mortality worldwide and the incidence of GC is most common

Correspondence to: Dr Yong Gao, Department of Oncology, Shanghai East Hospital, Tongji University School of Medicine, 150 Ji-Mo Road, Shanghai 200120, P.R. China

E-mail: gaoyon@hotmail.com

${ }^{*}$ Contributed equally

Key words: arginine/serine-rich coiled coil 1, gastric cancer, proliferation, migration, phosphatase and tensin homolog deleted on chromosome 10 in Asia, with Japan, Korea and China experiencing the highest number of cases of GC (1). The 5-year survival rate following diagnosis is $68.1 \%$ for localized cases, and 30.6 and $5.2 \%$ after the regional and distant spread of the disease, respectively (2). Unfortunately, patients with GC are usually diagnosed in the late stage with little scope for intervention (3). GC is multifactorial in development and genetics play an important role. A number of genes have been identified that exert effects on almost all aspects of cancer formation, ranging from tumorigenesis to metastasis (4), and thus therapeutic modalities and outcome are also influenced by these genes (5).

Arginine/serine-rich coiled coil 1 (RSRC1) encodes the protein of the arginine- and serine-rich family that plays an important role in cellular functions. The gene is located on chromosome 3 and its products play a pivotal role in constitutive and alternative mRNA splicing (6). RSRC1 plays an important role in the regulation of the transcription process (7) and studies to date have indicated that an absence of RSRC1 inhibits the second step of the splicing process. RSRC1 also regulates alternative splicing in a concentration-dependent manner (8). RSRC1 has been implicated in the pathogenesis of neuronal diseases, such as schizophrenia, as well as in certain types of cancer, such as glioblastoma (9) and prostate cancer (10). Another protein of the same family, RSRC2, is considered to play an important role in the development of esophageal carcinoma (11) and neuroblastoma (12). However, studies on the direct effects of RSRC1 on GC development are limited.

Phosphatase and tensin homolog deleted on chromosome 10 (PTEN) is a tumor suppressor gene with phosphatase-dependent and -independent activity (13). Its primary function is to regulate biological functions essential for genomic stability, survival, proliferation, migration and metabolism through the regression of the oncogenic PI3K/AKT signaling pathway (14). The strict control of PTEN expression through transcriptional, post-transcriptional and protein-protein interaction is essential for maintaining optimal cellular functionality $(15,16)$. PTEN inactivation or suppression through gene mutation, aberrant subcellular localization, or altered transcriptional and post-transcriptional regulation, which leads to tumor formation, occurs in various types of cancer, including GC (17). Previous research has suggested that PTEN expression is downregulated in GC and that the inactivation of PTEN causes accelerated tumor growth. For example, the overexpression of PTEN has been shown to inhibit the invasion and metastasis 
of GC via the downregulation of focal adhesion kinase (FAK) expression (18), and PTEN inactivation links the Hippo signaling pathway to the PI3K/AKT signaling pathway, thus potentiating tumorigenesis (19). In addition, some microRNAs (miRNAs or miRs) influence GC progression and outcome by altering PTEN function $(16,20)$.

In this study, we explored the role of RSRC1 in the development of GC. The frequent downregulation of RSRC1 expression was observed in GC tissues compared to adjacent normal tissues, suggesting that the lack of RSRC1 expression in GC cell lines could promote GC cell proliferation and migration. Furthermore, RSRC1 may exert its functions by regulating PTEN expression.

\section{Materials and methods}

Cell lines and culture conditions. The GC cell lines, SGC7901 and AGS, obtained from the Shanghai Cell Bank of the Chinese Academy of Sciences, were cultured in Dulbecco's modified Eagle's medium (DMEM, Corning, Inc.) supplemented with $10 \% \mathrm{FBS}$ and penicillin/streptomycin (M\&C Gene Technology Ltd.). The cells were incubated in an atmosphere of $5 \% \mathrm{CO}_{2}$ at $37^{\circ} \mathrm{C}$.

GC sample collection. GC tissues and paired adjacent normal tissues were collected from 36 patients diagnosed with GC at Shanghai East Hospital. Written informed consent was obtained from all the participants. All samples were flash-frozen in liquid nitrogen and stored at $-80^{\circ} \mathrm{C}$ prior to RNA extraction. This study was approved by the Ethics Committee of Shanghai East Hospital, Tongji University School of Medicine.

Immunohistochemical staining. Paraffin-embedded sections ( $4 \mu \mathrm{m})$ were prepared and processed for immunohistochemical staining. RSRC1 protein expression was assessed by immunohistochemical staining using an anti-RSRC1 antibody (\#23826-1-AP, Proteintech) at a dilution of 1:500, the sections were incubated at $4^{\circ} \mathrm{C}$ overnight. A two-step EnVision ${ }^{\mathrm{TM}} \mathrm{Kit}$ (\#K500711-2, Dako; Agilent Technologies, Inc.) was used following the manufacture's protocol to visualize positive staining. The results were classified as positively stained or negatively stained. The reviewing process was conducted in a blinded manner to prevent bias. An Olympus CX31 biological microscope (Olympus Corporation) was used to evaluate the staining results.

$R N A$ extraction and reverse transcription-quantitative $P C R$ $(R T-q P C R)$. Total RNA was extracted using TRIzol ragent (Sigma) and reverse transcription was performed using the commercial Primescript $^{\mathrm{TM}}$ RT Reagent kit with gDNA Eraser (Takara), following the manufacturer's instructions. The relative mRNA expression level of RSRC1 was determined by quantitative PCR (qPCR) with SYBR-Green reagent (Takara). The PCR thermocycling conditions were as the following parameters: $95^{\circ} \mathrm{C}$ for $1 \mathrm{~min}, 40$ cycles of $15 \mathrm{sec}$ at $95^{\circ} \mathrm{C}$ and $30 \mathrm{sec}$ at $60^{\circ} \mathrm{C}$. The relative mRNA expression level was calculated using the comparative $\Delta \Delta \mathrm{Cq}$ method (21), and $\beta$-actin was used as the endogenous control. Each measurement was carried out independently in triplicate. The following primers were used to amplify and measure the levels of RSRC1 and $\beta$-actin: RSRC1-qF,5-TCAAACGTGGGGAATCTGGA-3 and RSRC1-qR, 5-TGGCTTGGTCTTCCTCCTT-3; $\beta$-actin-qF, 5-CCTGGCACCCAGCACAATG-3 and $\beta$-actin-qR, 5-GGG CCGGACTCGTCATACT-3.

RNA interference and transfection. Cell transfection with small interference RNAs (siRNAs) was conducted using Lipofectamine 3000 (Invitrogen; Thermo Fisher Scientific) in accordance with the manufacturer's instructions. RSRC1-specific siRNAs (siRSRC1-1 and siRSRC1-2) were chemically synthesized (GenePharma). The sequences of siRSRC1-1 and siRSRC1-2 were as follows: sense, 5-GGU CGAGGGAAAUCCUAUA-3; sense, 5-GGGAUAGAGAAC GACGUAA-3. After 24-48 h post-transfection, the cells were subjected to subsequent experimentation.

Western blot analysis. Total proteins from were extracted from the cultured cells using RIPA buffer, and the supernatant was diluted in sodium dodecyl sulfate (SDS) loading buffer prior to storage. For western blot analysis, cell lysates $(25 \mu \mathrm{g}$ per lane) were electrophoresed by $10 \%$ sodium dodecyl sulfate-polyacrylamide gel electrophoresis (SDS-PAGE) and subsequently transferred onto polyvinylidene difluoride membranes (PVDF; Millipore). Protein concentration was determined using a BCA Protein Assay Kit (Thermo Fisher Scientific). The membranes were then blocked in non-fat milk for approximately $1 \mathrm{~h}$ at room temperature, followed by $2 \mathrm{~h}$ at room temperature or overnight at $4^{\circ} \mathrm{C}$ with the primary antibodies. After washing with phosphate-buffered saline (PBS) containing $0.05 \%$ Tween $^{\circledR} 20$ three times for $5 \mathrm{~min}$ each, the membranes were incubated with the secondary antibody [\#611-145-002, Rabbit IgG (H\&L) Antibody Dylight $^{\mathrm{TM}} 800$ Conjugated, 1:1,000; \#610-145-002, Mouse IgG (H\&L) Antibody DyLight ${ }^{\mathrm{TM}} 800$ Conjugated, 1:1,000, Rockland Immunochemicals, Inc.] for a further $1 \mathrm{~h}$ at room temperature. Protein detection was carried out using the Odyssey Infrared Imaging System (Li-COR Biosciences) according to manufacturer's instructions. The antibodies used in this study were as follows: RSRC1 (\#23826-1-AP, Proteintech, 1:500), $\beta$-actin (\#81178, Santa Cruz Biotechnology, 1:1,000), mammalian target of rapamycin (mTOR; \#2983, Cell Signaling Technology, 1:500), p53 (\#sc-47698, Santa Cruz Biotechnology, 1:500), PTEN (\#559600, BD Biosciences, 1:500), $\beta$-catenin (\#8480, 1:500), E-Cadherin (\#3195, 1:500), Snail (\#3879, 1:500) and zinc finger E-box-binding homeobox 1 (ZEB1; \#3396, 1:500) (all from Cell Signaling Technology) and FAK (\#66258-1-Ig, Proteintech, 1:500).

Cell proliferation assay. At $24 \mathrm{~h}$ following transfection, the cells $(3,000$ cells/well) were seeded in 96-well plates in triplicate and maintained in DMEM containing 10\% FBS for 5 days. Following the manufacturer's instructions, $10 \mu \mathrm{l}$ of Cell Counting kit- 8 reagent (CCK-8; Dojindo Laboratories) was added to each well for $1 \mathrm{~h}$ of incubation at $37^{\circ} \mathrm{C}$. The absorbance was read at a wavelength of $450 \mathrm{~nm}$ in an automated plate reader (SpectraMax M5, Molecular Devices, LLC). All experiments were independently repeated at least 3 times.

Cell migration assay. A 24-well Transwell chamber (pore size, $8 \mu \mathrm{m}$; Costar; Corning, Inc.) was used to perform cell migration assay. Briefly, cells were harvested and suspended in 


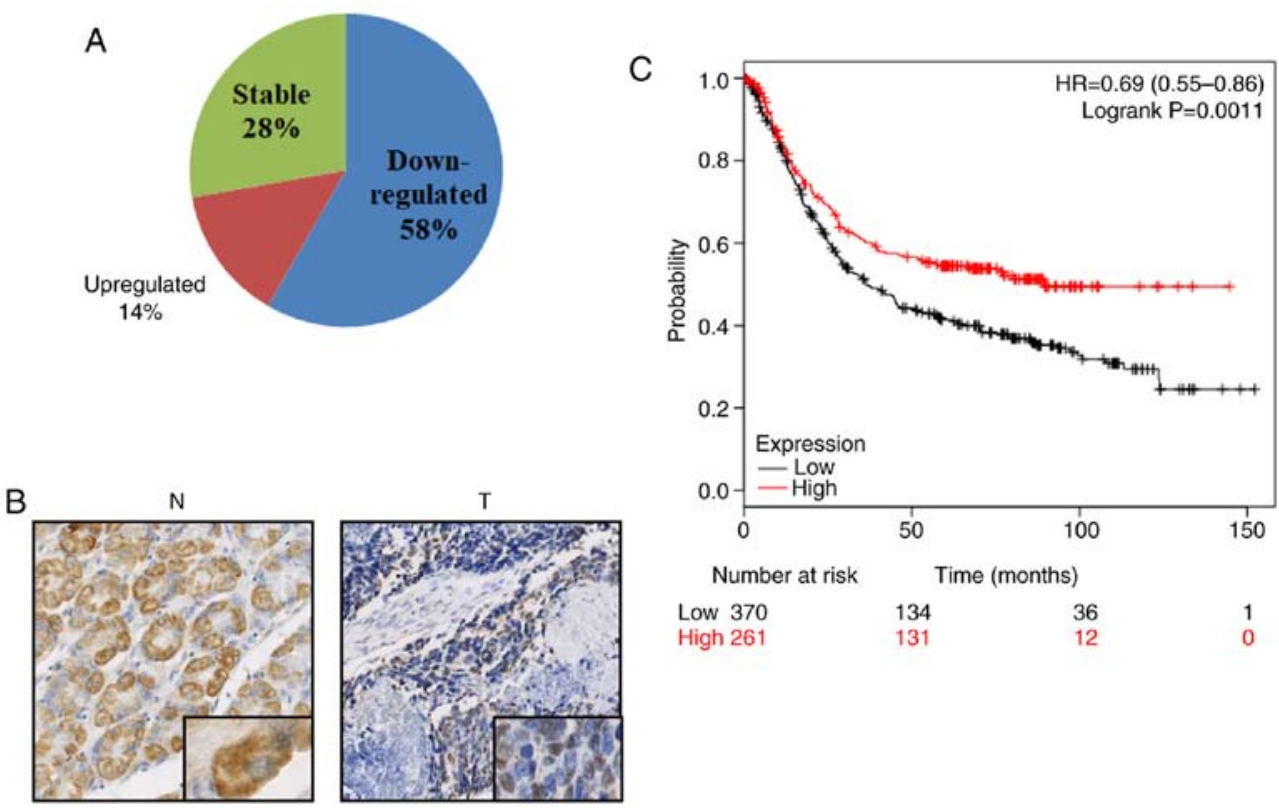

Figure 1. Downregulation of RSRC1 is associated with a poor prognosis of patients with GC. (A) Pie chart showing that RSRC1 expression is downregulated in the majority of the tumor tissues compared to the paired adjacent normal tissues. According to the fold change (T/N) of RSRC1 mRNA expression in each pair of samples, the results of RT-qPCR were classified as upregulated $(>2.0)$, stable $(0.5-2.0)$ and downregulated $(<0.5)$. (B) Representative images of immunohistochemistry showing the protein expression of RSRC1 in normal tissues compared to tumor tissues. Magnification, x40 (insert) and x200. (C) Kaplan Meier curves comparing the overall survival (OS) of patients with tumors with a low expression of RSRC1 (black line; $\mathrm{n}=370$ ) and a high RSRC1 expression (red line; $\mathrm{n}=261$ ), $\mathrm{P}<0.01, \log$-rank test. T, tumor tissue; $\mathrm{N}$, adjacent normal tissue; RSRC1, arginine/serine-rich coiled coil 1; GC, gastric cancer.

DMEM without FBS at a density of $1 \times 10^{5}$ cells $/ \mathrm{ml}$ at $48 \mathrm{~h}$ after transfection with siRNAs, and $400 \mu$ l of the cell suspension was added into the upper chamber, while $800 \mu 1$ DMEM containing $10 \%$ FBS was loaded in the bottom chamber. After incubation for $24 \mathrm{~h}$ at $37^{\circ} \mathrm{C}$, the non-migrating cells in the upper chamber were removed with a cotton swab, and the migrated cells on the bottom surface of the filter were fixed in $4 \%$ paraformaldehyde for $5 \mathrm{~min}$ at room temperature, then stained with $0.5 \%$ crystal violet at room temperature (\#C3886, Sigma-Aldrich; Merck $\mathrm{KGaA}$ ) for $10 \mathrm{~min}$ and counted under a phase contrast microscope (Leica DM6000B, Leica Microsystems, Inc.) in five randomly selected fields at a magnification of x200.

EdU labeling and immunofluorescence. The cells were seeded in a 24-well culture plates and $24 \mathrm{~h}$ later were incubated with 50 mM 5-ethynyl-2'-deoxyuridine (EdU; Guangzhou RiboBio Co., Ltd.) for $2 \mathrm{~h}$ at room temperature. They were then stained with Apollo 567 for $30 \mathrm{~min}$ at room temperature away from light as per the manufacturer's protocols (Guangzhou RiboBio Co., Ltd.) stained cells were observed and counted under a microscope (Leica DMI3000B, Leica Microsystems, Inc.). All experiments were repeated at least 3 times independently.

Statistical analysis. Quantitative values are represented as the means \pm SD. Analyses were done using GraphPad Prism software 7.0 (GraphPad Software, Inc., San Diego, CA, USA). Statistical significance for quantitative data was determined by one-way analysis of variance (one-way ANOVA) and Dunnett's multiple comparisons test was used as a post hoc test. The Kaplan-Meier and log-rank tests were used for the overall survival analysis from Kaplan-Meier Plotter datasets (http://kmplot.com/analysis/). $\mathrm{P}<0.05$ was considered to indicate a statistically significant difference.

\section{Results}

RSRC1 expression is frequently downregulated in GC. To examine the expression of RSRC1 in GC, paired tumor tissues and adjacent normal tissues were collected from 36 patients with GC, and RT-qPCR analysis was conducted. As shown in Fig. 1A, RSRC1 mRNA expression was significantly downregulated in $21 / 36(58 \%)$ tumor tissues compared with paired normal tissues; 10/36 (28\%) of the GC specimens exhibited no significant difference in RSRC1 mRNA expression between the tumor tissues and paired normal tissues, and only 5/36 (14\%) of the GC specimens exhibited an upregulated RSRC1 mRNA expression in the tumor tissues. To confirm the results of RT-qPCR, 5 GC tumor and normal tissue pairs were randomly selected from the collected samples and immunohistochemical staining analysis with specific antibody directed against RSRC1 was performed. Representative images are presented in Fig. 1B, which indicated the reduced protein expression of RSRC1 in the GC tumor tissues. Furthermore, survival analysis obtained from the Kaplan-Meier Plotter datasets demonstrated that a reduced expression of RSRC1 was associated with a worse overall survival rate of patients with GC (Fig. 1C). Collectively, these results indicate a strong association between RSRC1 and GC.

RSRC1 suppresses the proliferative ability of $G C$ cells. Given that RSRC1 is downregulated in GC, we then explored its function in GC progression. Synthesized siRNAs against RSRC1 (siRSRC1-1 and siRSRC1-2) were transiently transfected into the AGS and SGC7901 cells and western blot analysis was performed to validate the knockdown effects (Fig. 2A). CCK-8 assays were deployed and cell growth curves indicated that the cells transfected with siRSRC1-1 and siRSRC1-2 had 

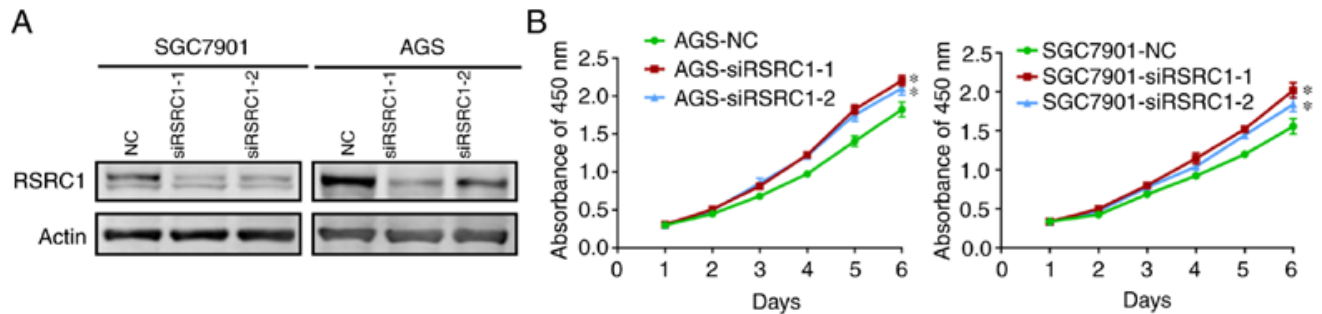

C
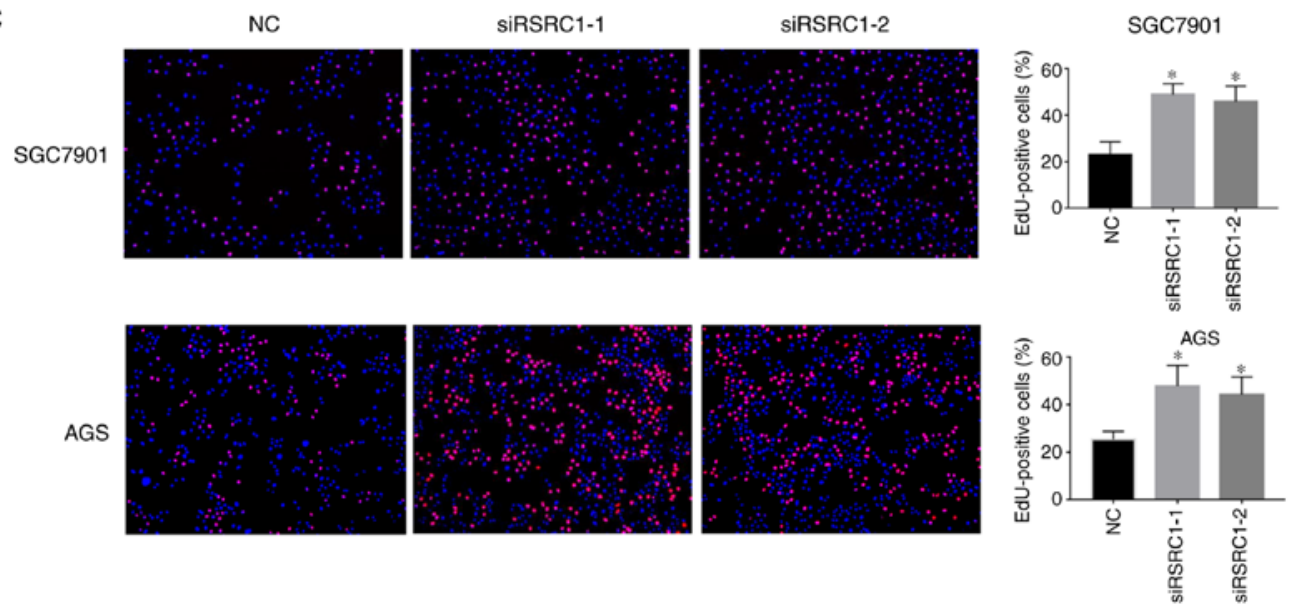

Figure 2. RSRC1 suppresses GC cell proliferative ability. (A) RSRC1 knockdown in GC cell lines SGC7901 and AGS was carried out by transfection with small interference RNAs (siRNAs) si-RSRC1-1 and si-RSRC1-2, and the knockdown efficiency was confirmed by western blot analysis. (B) Cell growth curves following RSRC1 knockdown in SGC7901 and AGS cells showing accelerated growth rates as compared to normal controls (NC). (C) EdU incorporation assay was performed to compare cell proliferation ability of SGC7901 and AGS cells following knockdown of RSRC1 and their corresponding controls. Magnification, $x 10$. Data are presented as the means \pm SD of 3 independent experiments, "P<0.05 vs. NC. RSRC1, arginine/serine-rich coiled coil 1; GC, gastric cancer.

significantly higher growth rates than those transfected with siNC (Fig. 2B). Moreover, EdU incorporation assays were conducted to further confirm the role of RSRC1 in GC cell proliferation, and we found that RSRC1 knockdown resulted in an increase in the percentage of EdU-positive cells as compared to the controls (Fig. 2C). Taken together, these data provide evidence that RSRC1 suppresses GC cell proliferation.

RSRC1 knockdown enhances the migratory ability of GC cells. Since metastasis plays a key role in the poor prognosis of patients with GC, we then examined the effect of RSRC1 on the migratory ability of GC cells. After knocking down RSRC1 expression in AGS and SGC7901 cells, Transwell cell migration assays were employed, and results are presented in Fig. 3. The cells transfected with siRSRC1-1 and siRSRC1-2 exhibigted significantly higher numbers of migrated cells, suggesting a negative role of RSRC1 in GC cell migration. Therefore, our observations illustrate the importance of RSRC1 in suppressing GC cell proliferation and migration.

RSRC1 regulates the expression of PTEN. To identify the molecular mechanisms through which RSRC1 inhibits the proliferation and migration of GC cells, we performed western blot analysis to investigate the expression of specific proteins related to tumor proliferation, cell apoptosis and epithelial-mesenchymal transition (EMT) after RSRC1 expression was silenced in the AGS cells. The results indicated that PTEN expression was suppressed and FAK expression was enhanced following the knockdown of RSRC1 in GC cells (Fig. 4A). PTEN is a well-known tumor suppressor gene in different cancer types, and PTEN has been previously reported to negatively regulate FAK expression in GC (18), suggesting that RSRC1 suppresses cell proliferation and migration by regulating PTEN expression in GC cells. Thus, we performed RT-PCR to investigate whether RSRC1 regulates PTEN expression at the transcriptional level. The changes in the mRNA expression of PTEN were not obvious after the silencing of RSRC1 expression in GC cells (Fig. 4B), suggesting that the regulation of PTEN expression by RSRC1 does not occur at the level of transcription. In summary, the data presented herein provide a possible mechanism through which RSRC1 inhibits GC cell proliferation and migration.

\section{Discussion}

GC is a leading cause cancer related-mortality worldwide and is multifactorial in its development, with genetics playing an important role. However, further research concerning the tumorigenesis of GC is required in order to improve the early diagnosis and treatment modalities of GC. To the best of our knowledge, the present study demonstrated for the first time that RSRC1, which plays a pivotal role in the constitutive and alternating splicing of the pre-mRNA, is a potential tumor suppressor in GC.

From the clinical samples, we found that RSRC1 expression was frequently downregulated in GC tissues, and survival analysis from Kaplan-Meier plot datasets revealed that RSRC1 was associated with the prognosis of patients with 
A

SGC7901

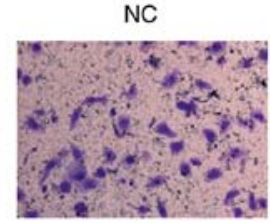

AGS

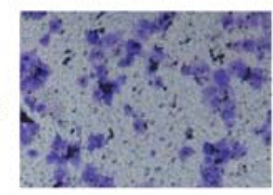

siRSRC1-1
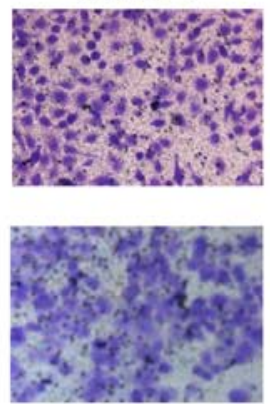

SiRSRC1-2
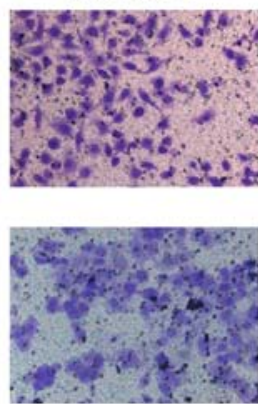

B

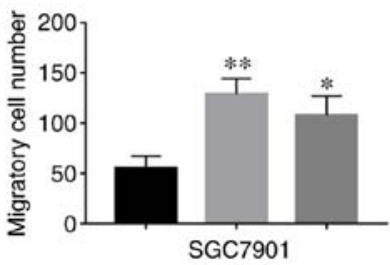

SGC7901-NC

SGC7901-siRSRC1-1

SGC7901-siRSRC1-2

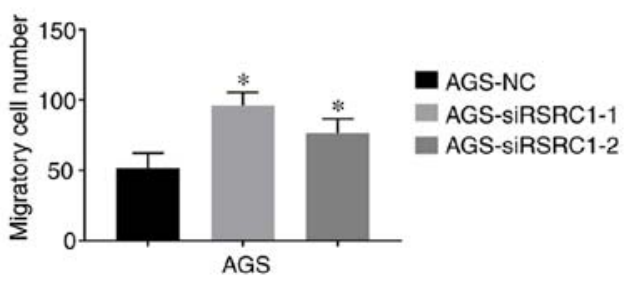

Figure 3. Knockdown of RSRC1 promotes GC cell migration. (A) Cell migratory ability was measured by Transwell migration assays following transfection. Representative images of migrated cells are shown, and all the experiments were repeated at least 3 times independently. Magnification, x100. (B) The number of migrated cells was counted in 5 randomly selected microscopic fields and the data are presented as the means $\pm \mathrm{SD}$. ${ }^{*} \mathrm{P}<0.05$ and ${ }^{* *} \mathrm{P}<0.01$ vs. NC group. RSRC1, arginine/serine-rich coiled coil 1; GC, gastric cancer.

A

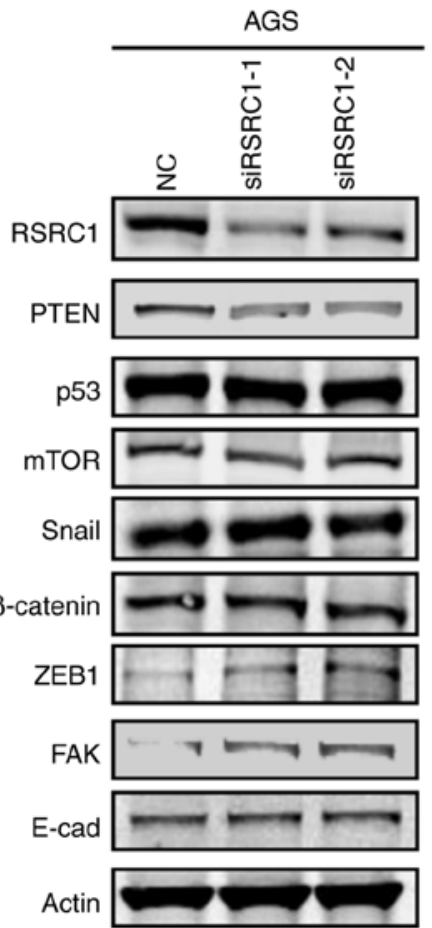

B
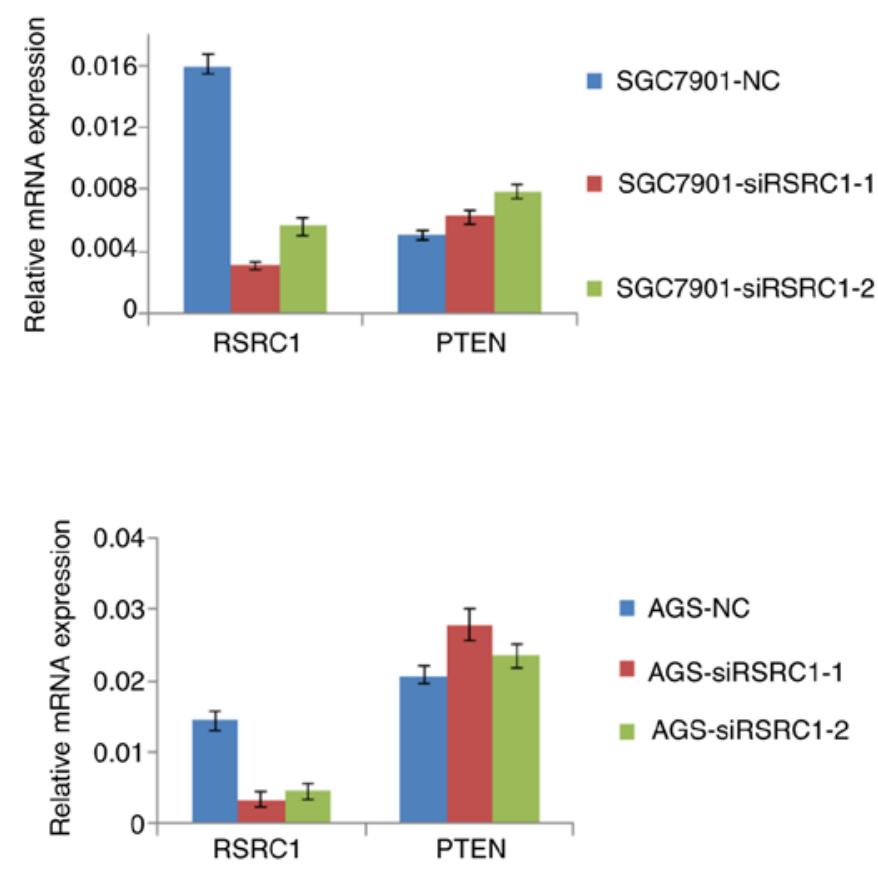

- AGS-NC

- AGS-siRSRC1-1

aGS-siRSRC1-2

Figure 4. RSRC1 regulates PTEN expression in GC cells. (A) Western blot analysis was performed to detect the expression levels of specific proteins involved in signaling pathways related to tumor development after the silencing of RSRC1 expression in AGS cells. (B) RT-qPCR was performed following RSRC1 knockdown in SGC7901 and AGS cell lines to observe the alterations in PTEN mRNA expression. RSRC1, arginine/serine-rich coiled coil 1; GC, gastric cancer.

GC. CCK-8 assays and EdU incorporation assays revealed that the silencing of RSRC1 expression in GC cells promoted cell growth and proliferation. The results of Transwell migration assays further indicated that the knockdown of RSRC1 promoted the migratory capacity of GC cells. These observations provided a strong basis for the consideration of RSRC1 as a tumor suppressor gene in GC development.
RSRC1 mainly controls the second step of the splicing process (8). It is also implicated in the regulation of the transcription process through ERB repression by SUMOylation mediated by RSRC1 (22). The single nucleotide polymorphism (SNP) of RSRC1 has been found in schizophrenia and the downregulation of RSRC1 has been found in dementia and Alzheimer's disease (7). RSRC1 polymorphism increases the 
susceptibility of children to neuroblastoma (23). However, its role in the development of $\mathrm{GC}$ has not yet been elucidated. This study initially explored the role of RSRC1 in GC development; however, whether its effects on GC cell proliferation are associated with the alternative splicing of mRNAs, remains to be further investigated.

PTEN is one of the most frequently mutated tumor suppressor genes and has been widely studied in different types of human malignancies. A number of studies on GC have demonstrated that PTEN inhibits tumor progression via the negative regulation of the PI3K signaling pathway and its downstream components (24-26) and the AKT/GSK3 $\beta$ signaling pathway (27). PTEN has also been found to negatively regulate the expression of the well-known oncogene, FAK (18). In this study, we found that RSRC1 suppressed GC cell proliferation and migration, possibly by regulating PTEN expression, and this regulation did not occur at the transcriptional level. Hence, in future studies, further experiments are required at both the cellular and molecular levels to confirm whether RSRC1 functions are dependent on the regulation of PTEN expression and whether this type of regulation is related to mRNA alternative splicing.

In conclusion, this study identified RSRC1 as a potential tumor suppressor gene in GC. The reduced expression of RSRC1 was associated with a poorer prognosis of patients with GC; RSRC1 suppressed GC cell proliferation and migration possibly by regulating the PTEN/FAK signaling pathway. Our findings provide novel insight into the mechanisms of tumorigenesis and development of GC.

\section{Acknowledgements}

Not applicable.

\section{Funding}

This study was supported by grants from the National Key Research and Development Program of China (grant no. 2017YFC1308900), National Natural Science Foundation of China (grant no. 81472576 and 81502043) and Key Disciplines Group Construction Project of Pudong Health Bureau of Shanghai (grant no. PWZxq2017-13).

\section{Availability of data and materials}

All data generated or analyzed during this study are included in this published article.

\section{Authors' contributions}

YG designed the study; SY, NG and MQ performed the experiments, SY and MQ processed the figures. NG and MQ performed statistical analysis. SY, NG and YG were involved in the writing and revising of the manuscript.All authors read and approved the final manuscript.

\section{Ethics approval and consent to participate}

The experiments were approved by the Ethics Committee of Shanghai East Hospital, Tongji University School of Medicine.
Informed consent was obtained from all patients prior to sample collection.

\section{Patient consent for publication}

Not applicable.

\section{Competing interests}

The authors declare that they have no competing interests.

\section{References}

1. Carcas LP: Gastric cancer review. J Carcinog 13: 14, 2014.

2. Watson GL, Goff C, and Shi P: Pohlmann: Molecular profiling of gastric cancer. My Cancer Genome: Mar 16, 2018.

3. Mihmanli M, Ilhan E, Idiz UO, Alemdar A and Demir U: Recent developments and innovations in gastric cancer. World J Gastroenterol 22: 4307-4320, 2016.

4. McFarlane M, Brettschneider J, Gelsthorpe A, James S, Snead D, Gopalakrishnan K, Mehenna H, Jankowski J, Arasaradnam R and Nwokolo C: An assessment of candidate genes to assist prognosis in gastric cancer. J Gastrointest Oncol 9: 303-310, 2018.

5. Yusefi AR, Bagheri Lankarani K, Bastani P, Radinmanesh M and Kavosi Z: Risk factors for gastric cancer: A systematic review. Asian Pac J Cancer Prev 19: 591-603, 2018.

6. McDaniel LD, Conkrite KL, Chang X, Capasso M, Vaksman Z, Oldridge DA, Zachariou A, Horn M, Diamond M, Hou C, et al: Common variants upstream of MLF1 at 3q25 and within CPZ at 4p16 associated with neuroblastoma. PLoS Genet 13: e1006787, 2017.

7. Perez Y, Menascu S, Cohen I, Kadir R, Basha O, Shorer Z, Romi H, Meiri G, Rabinski T, Ofir R, et al: RSRC1 mutation affects intellect and behaviour through aberrant splicing and transcription, downregulating IGFBP3. Brain 141: 961-970, 2018.

8. Cazalla D, Newton K and Caceres JF: A novel SR-related protein is required for the second step of Pre-mRNA splicing. Mol Cell Biol 25: 2969-2980, 2005.

9. Teplyuk NM, Uhlmann EJ, Gabriely G, Volfovsky N, Wang Y, Teng J, Karmali P, Marcusson E, Peter M, Mohan A, et al: Therapeutic potential of targeting microRNA-10b in established intracranial glioblastoma: First steps toward the clinic. EMBO Mol Med 8: 268-287, 2016.

10. Pflueger D, Terry S, Sboner A, Habegger L, Esgueva R, Lin PC, Svensson MA, Kitabayashi N, Moss BJ, MacDonald TY, et al: Discovery of non-ETS gene fusions in human prostate cancer using next-generation RNA sequencing. Genome Res 21: 56-67, 2011.

11. Kurehara H, Ishiguro H, Kimura M, Mitsui A, Ando T, Sugito N, Mori R, Takashima N, Ogawa R, Fujii Y and Kuwabara Y: A novel gene, RSRC2, inhibits cell proliferation and affects survival in esophageal cancer patients. Int J Oncol 30: 421-428, 2007.

12. Wolf M, Korja M, Karhu R, Edgren H, Kilpinen S, Ojala K, Mousses S, Kallioniemi A and Haapasalo H: Array-based gene expression, CGH and tissue data defines a 12 q24 gain in neuroblastic tumors with prognostic implication. BMC Cancer 10: 181, 2010.

13. Hopkins BD, Hodakoski C, Barrows D, Mense SM and Parsons RE: PTEN function: The long and the short of it. Trends Biochem Sci 39: 183-190, 2014.

14. Yao H, Su S, Xia D, Wang M, Li Z, Chen W, Ren L and Xu L: F-box and leucine-rich repeat protein 5 promotes colon cancer progression by modulating PTEN/PI3K/AKT signaling pathway. Biomed Pharmacother 107: 1712-1719, 2018.

15. Naderali E, Khaki AA, Rad JS, Ali-Hemmati A, Rahmati M and Charoudeh HN: Regulation and modulation of PTEN activity. Mol Biol Rep 45: 2869-2881, 2018.

16. Lu R, Zhao G, Yang Y, Jiang Z, Cai J, Zhang Z and Hu H: Long noncoding RNA HOTAIRM1 inhibits cell progression by regulating miR-17-5p/PTEN axis in gastric cancer. J Cell Biochem 120: 4952-4965, 2019.

17. Lee YR, Chen M and Pandolfi PP: The functions and regulation of the PTEN tumour suppressor: New modes and prospects. Nat Rev Mol Cell Biol 19: 547-562, 2018. 
18. Zhang LL, Liu J, Lei S, Zhang J, Zhou W and Yu HG: PTEN inhibits the invasion and metastasis of gastric cancer via downregulation of FAK expression. Cell Signal 26: 1011-1020, 2014.

19. Xu W, Yang Z, Xie C, Zhu Y, Shu X, Zhang Z, Li N, Chai N, Zhang S, Wu K, et al: PTEN lipid phosphatase inactivation links the hippo and PI3K/Akt pathways to induce gastric tumorigenesis. J Exp Clin Cancer Res 37: 198, 2018.

20. Ding K, Wu Z, Wang N, Wang X, Wang Y, Qian P, Meng G and Tan S: MiR-26a performs converse roles in proliferation and metastasis of different gastric cancer cells via regulating of PTEN expression. Pathol Res Pract 213: 467-475, 2017.

21. Livak KJ and Schmittgen TD: Analysis of relative gene expression data using real-time quantitative PCR and the 2(-Delta Delta C(T)) method. Methods 25: 402-408, 2001

22. Chen L, Li W, Qiu W, Ren W, Li Q, Han B, Zhou L, Cheng L, Zhang Hand Ye Q: RSRC1 SUMOylation enhances SUMOylation and inhibits transcriptional activity of estrogen receptor $\beta$. FEBS Lett 589: 1476-1484, 2015.

23. Tang J, Liu W, Zhu J, Zhang J, Wang FH, Liang JH, Zeng JH, Wang $\mathrm{H}$, Xia $\mathrm{H}$ and He J: RSRC1 and CPZ gene polymorphisms with neuroblastoma susceptibility in Chinese children. Gene 662: 83-87, 2018.
24. Davidson L, Maccario H, Perera NM, Yang X, Spinelli L, Tibarewal P, Glancy B, Gray A, Weijer CJ, Downes CP and Leslie NR: Suppression of cellular proliferation and invasion by the concerted lipid and protein phosphatase activities of PTEN. Oncogene 29: 687-697, 2010.

25. Ko BS, Chang TC, Chen CH, Liu CC, Kuo CC, Hsu C, Shen YC, Shen TL, Golubovskaya VM, Chang CC, et al: Bortezomib suppresses focal adhesion kinase expression via interrupting nuclear factor-kappa B. Life Sci 86: 199-206, 2010.

26. Wang $X$ and Jiang X: PTEN: A default gate-keeping tumor suppressor with a versatile tail. Cell Res 18: 807-816, 2008

27. Ma J, Guo X, Zhang J, Wu D, Hu X, Li J, Lan Q, Liu Y and Dong W: PTEN gene induces cell invasion and migration via regulating AKT/GSK-3 $\beta / \beta$-catenin signaling pathway in human gastric cancer. Dig Dis Sci 62: 3415-3425, 2017.

(i) (9) This work is licensed under a Creative Commons Attribution-NonCommercial-NoDerivatives 4.0 International (CC BY-NC-ND 4.0) License. 